THE LANCET, December 30, 1905.

\section{A ingerture}

\author{
ON
}

SOME OF THE CLINICAL ASPECTS OF
PNEUMONIA.

Being one of a Series of Lectures delivered at the West London Hospital to Post-Graduates on May 30th, 1905,

\section{By DONALD W. C. HOOD, C.V.O., M.D. Cantab., F.R.C.P. Lond.,}

SENIOR PHYSICIAN TO THE HOSPITAL.

GENTLEMEN, - During the past few months we have had many opportunities of watching the various conditions and symptoms with which pneumonia may be connected and complicated. It will be to our mutual advantage to group together some of these cases and to consider them more in detail than is possible at our bedside demonstrations.

Pneumonia, as you are all well aware, is described as being of one of two principal forms. We are accustomed to classify the disease as belonging to either the so-called lobar or lobular type, the later variety being as commonly known under the name catarrhal. Until but lately we have been taught that the pathology of each of these forms of pneumonia was essentially different. There cannot be a doubt that in ordinary circumstances the one form is sharply separated from the other and in 99 cases out of a 100 when the symptoms are compared together they are of a totally different character. Although the question of the absolute distinction in pathology may be said to be still sub judice the researches of the students of bacteriology have, I think, almost conclusively proved that the pneumococcus may be the essential cause of both conditions of pneumonic inflammation and that the micro-organism may, under possibly different environment, give rise to both catarrhal and lobar pneumonia. Clinical and bacteriological evidence at the present time insists that we should include under conditions of pneumonia another class, happily less frequent than either of the two so usually met with, and we have to recognise a state of what may well be called "pneumonic fever," a form of rapid and disastrous infection, not only of the pulmonary area, but of other parts of the body-to wit, the endocardium, pericardium, and joints. I have before me the notes of an interesting case published in the British Medical Journal ${ }^{1}$ by Dr. G. Newton Pitt. They relate to a young man, aged 17 years, who was admitted into hospital for symptoms of intense pulmonary mischief of acute type, but excepting a slight pleuritic rul without appreciable local symptoms. The diagnosis was doubtful, the history was vague, and the patient was unable to answer questions. The case proved to be one typical of pneumococcal septicæmia, in which the poison overwhelmed the patient before there was time for consolidation of the lung to occur. The diagnosis in these extreme cases is one of great difficulty. The main point to bear in mind is the rapid spreading of infection from organ to organ: the lung, pleura, heart, meninges, joints, and colon may be almost simultaneously affected. Such cases might be justly called "pneumonic fever."

But, gentlemen, with these few remarks on the underlying pathology of pneumonia I will dismiss the subject and $I$ ask your attention to some of the symptoms of the many various forms and degrees of the pneumonic process. At the bedside we have abundant oppor tunity for observing the varying phases of this common disease. I will ask you to permit me to use the word pneumonia in the widest generic sense. The attempt will be made to discuss some of the following varieties often giving rise to vastly differing symptoms pneumonia of the apex and at the base; pneumonia with symptoms directly referable to the abdominal area-a most important group ; the disseminated, migrating, or congestive type, as seen in influenza ; the senile and rapidly infiltrating form; hæmorrhagic pneumonia with profuse hæmoptysis;

No. 4296. the pneumonia of cerebral type, and that form so often perplexing to us who have been watching the case for daysthe deep-seated or concealed-in which we have all the clinical phenomena of a pulmonary attack and yet the physical conditions are at first absent, appearing only after several days of illness.

It is of importance to remember that the primary anatomical position of the commencing pneumonia may profoundly influence the symptoms of the attack. Thus in a certain proportion of patients suffering from pneumonia of the apex constitutional disturbances are apt to arise. These may easily mislead us as to the true nature of the illness from which the patient is suffering, the symptoms, especially with children, being of cerebral type, headache, intolerance of light, extreme delirium, and restlessness. And, again, in some patients suffering from pneumonia of one or other base the constitutional disturbance induced by the attack may as closely simulate functional mischief of either liver or stomach, and we may see a state of gastritis or jaundice induced by inflammation above the diaphragm. Within the past few days I saw a lady in the country who was suffering from pneumonia of the base of the right lung. She had been jaundiced at the commencement of her illness six weeks previously to my visit. A diagnosis of inflammation of the liver had been made! I will ask your attention to a few of these cases; they are of much interest and worthy of your earnest consideration.

\section{Cases of Pulmonary Disease in which the Primary}

Symptoms aRe THOSE OF ABdominal Disease.

Clinically it is of the utmost importance to bear in mind that the initial onset, the primary symptoms of an attack of pneumonia, may be pain, often very severe, referred to some part of the abdomen. In a lecture given in this hospital in August, 1894, I spoke of these cases and stated that when pneumonia commences in the lower lobe of either lung the inflammatory process is frequently connected with symptoms which may easily put us off our guard. We. may, in such circumstances, very readily fall iato errorand possibly overlook the true significance of the primary symptoms. A delicate lad was seen by me. He had during the night been suddenly seized with severe pain over the left epigastric region. The pain was accompanied with vomiting which had been incessant for several hours. There was a temperature of $102^{\circ} \mathrm{F}$, and the breathing was quick. During the first 24 hours the stomach irritation was practically the only symptom, but the following day sharp. crepitant râles at the base of the left lung, followed by consolidation, established the true nature of the illness. During the past ten years I have seen many cases of this class and I think that they are all the more worthy of our careful attention inasmuch as with some abdominal pain is almost a warrant for operation at sight.

A few months ago a young man was admitted under my care as a case of abdominal disease. The patient had been sent to the hospital supposed to be suffering from acute ptomaine poisoning, the prominent symptoms being vomiting and some abdominal pain, referred to the splenic area and passing to the umbilicus. Over this region there was extreme. tenderness. The pain, however, was more superficial than deep. On attracting the patient's attention firm, deep pressure could be made without causing any distress. The temperature was raised and the respirations were over 40 . On examining the chest tubular breathing could be heard at the extreme left base. The case ran an ordinary course of lobar pneumonia. About the same time there was another patient in. hospital under my care whose symptoms gave rise to a good deal of anxiety. He was a man of middle age, admitted as suffering from abdominal disease, and many who saw him during the first 48 hours afrer admission considered that the abdomen was the seat of disease. The balance of clinical evidence was against such a view. There was no vomiting or diarrhoea. The abdomen. was uniformly much distended and tympanitic. The temperature was high, being $104^{\circ} \mathrm{F}$., the respiration was rapid, and the pulse was quick and sharp. The man was noisy with delirium. On making a careful examination of the chest some sharp crepitation could be detected at the extreme base of the right lung. Consolidation and tubular breathing rapidly supervened and the distension and pain. of the abdomen gradually became less and the symptoms of illness were those of an ordinary uncomplicated lobar pneumonia. In these cases the abdominal symptoms were in strong evidence ; I might say that they were paramount. 
Clinical medicine enforces the fact that pneumonia and pleurisy may very closely, at the initial stage, simulate acute gastric irritation, hepatic disorders, appendicitis, renal calculus, or peritonitis.

A case I saw some years ago with the late Mr. J. L. Propert impressed me much with regard to the nerve-supply of the abdominal area when influenced by commencing disease within the thorax. The patient was a lady of advanced life who had been ill for only a short time, suffering from agonising pain referred to the right side of the abdomen. She had been seen by several medical men. One considered that the pain was renal while another thought that it was due to the appendix. Pain, and that of a very severe character, was the only symptom. There was no pyrexia nor was there any sign of inflammatory disease. At the time of my visit the severity of the pain was very great. It was scarcely controlled by subcutaneous injections of morphine. On carefully examining the chest I considered that there was a very slight effusion in the right chest. I could detect nothing more. I saw the patient again in two days' time. The effusion in the right pleura was now definite. There was still no other symptom than pain. The cause was certainly not acute pleurisy. The absence of any pyrexia rendered such inflammatory lesion improbable. There was no indication of a gastric ulcer perforating into the chest, cases which are often accompanied by complex equivocal symptoms. Three main factors only were present to enable us to solve a problem of the greatest difficulty: pain of the severest character radiating over the right side of the abdomen, a slight effusion into the right chest without any symptoms of acute mischief, and the age of the patient. In my opinion the probable cause of trouble was malignant disease. Within a few days the diacrnosis was verified and a small malignant growth was found implicating the lower dorsal nerves as they issued from the spinal column.

Excessive irritability of the stomach with a high teniperature should always draw attention to the chest and thus save the mistake being made of looking upon an oncoming pneumonia as being acute gastritis. I saw with Sir Frederick Treves a young girl on whom he had operated ten days previously for appendicitis. There had been no untoward symptoms. The wound was perfectly healthy. On the eighth or ninth day after the operation the patient was seized suddenly with illness of an indefinite nature, the principal symptom being extreme irritability of the stomach. No food could be retained and incessant attacks of vomiting were rapidly sapping the strength of the patient. 48 hours after the commencement of illness symptoms developed at the base of the left lung. There were a slight cough and quickened inspiration. The cough increased, becoming paroxysmal. During a severe paroxysm a few ounces of pus were ejected and from this moment all symptoms subsided and the patient made a complete recovery. I have only alluded to the salient points in this case, but it was a good example of pulmonary trouble producing symptoms of extreme gastric irritation.

A commencing pneumonia may cause a localised abdominal pain difficult to distinguish from the pain at the commencement of appendicitis. Without a practical experience of such cases it would seem almost impossible to confound two such widely differing acute diseases, but the abdomen has been not infrequently opened on account of abdominal pain which has been caused by an inflammatory lesion commencing within the chest. The following is a striking example of this unusual form of commencing pleuropneumonia. A young married woman, living in the north of England, after a long day's hunting experienced pain, or rather discomfort, on the right side of the abdomen. She thought it was due to strain. The following day she travelled to London. The pain much increased in severity. I saw her at eight o'clock in the evening, at which time the pain in the abdomen was very severe. It was absolutely limited to the right side and the maximum intensity was over the ileo-cæcal region. At this part of the abdomen the superficial tenderness was acute, but deep pressure could be fairly well borne on engaging the attention of the patient. Breathing was shallow and rapid, deep respirations increasing the pain in the abdomen. There was pyrexia, the temperature being $102.4^{\circ} \mathrm{F}$., and the pulse 104 . Four hours later the pain was of extreme severity. The temperature had risen to $104 \cdot 5^{\circ}$, and at the time I could detect some sharp crepitant râles at the extreme base of the right lung. 'The abdominal pain quickly subsided and the case assumed all the ordinary characters of a sharp pleuritis which was followed by a slight effusion. I must confess that when I first saw this patient I thought that she was suffering from an acute attack of local peritonitis, the symptoms being limited to the abdomen, and I could detect no sign of chest mischief. It was an interesting and important fact that within six hours the aspect of the case had entirely changed,

From my own experience I consider that at the commencement of such an illness as I have just referred to it is impossible to make a certain diagnosis. There are a few main factors which should be carefully remembered; they will assist us in distinguishing between the acute abdominal seizure and that of commencing inflammatory mischief within the thorax. The principal symptom, to which the utmost care should be given, is the rate of respiration. In all chest cases the rapidity of respiration is much increased. Of course, this may also be the case with acute abdominal seizures but with the latter the temperature and pulse are usually lower. With a marked pyrexia, by which I mean a temperature of at least $102^{\circ} \mathrm{F}$., a quick, sharp pulse and respirations of from 30 to 40 , and a severe abdominal pain which is accompanied with distinct hyperæsthesia of the skin, so much so that the patient complains of a mere touch on the surface, the chances are in favour of intrathoracic mischief, and at any rate bearing such conditions well in mind will tend to prevent our falling into error. But a few weeks ago one of our resident medical officers telephoned to me about a child who had been admitted to hospital during the evening as a case of abdominal disease, the symptoms being a painful distended abdomen. I inquired the rate of the respirations it was 48. On this information I suggested waiting before calling in surgical help. Basic pneumonia of a very severe type rapidly developed.

In October, 1903, we had a very interesting case in our wards emphasising the importance of recognising the fact that severe abdominal pain may be due to disease of the chest. A young well-nourished man was admitted with the symptoms of an ordinary left lobar pneumonia. On the third day after admission he was suddenly seized with pain referred to the epigastrium. His breathing was affected and the symptoms, although complicated by the extreme colic, were distinctly those of the chest. Within 24 hours of the first complaint of pain there were physical signs at the base of the right lung, and the abdominal pain, which had evidently been due to the implication of the diaphragmatic pleura, gradually subsided. At the beginning of the year I saw in the country a patient suffering from acute gastrio irritation which had been present for rather more than 48 hours. There were much abdominal pain and general gastric disturbance. There were symptoms of commencing pneumonia which rapidly developed as the gastric irritation passed away.

A few days ago I saw with Dr. C. G. May a patient, a lady in whom the prominent feature of the illness was extreme pain referred to the right ileo-cæcal region. The pain had lasted for some days; there had been some rise of temperature but practically none of the ordinary symptoms of chest disease. Over the ileo-cæcal region the pain was complained of as being very severe. A carefu examination failed to find any abdominal cause for this pain. In the chest at the posterior part of the right side, over a very limited area, distinct breath ing of a tubular note could be heard. Within 36 hours the abdominal pain subsided and the conditions assumed those of pneumonia.

In the following case an attack of diaphragmatic pleurisy, with pneumonia of the extreme base of the right lung, closely simulated renal colic, of which the patient had experienced several seizures. The character of the pain, from the patient's point of view, was exactly the same as when he had passed renal calculi on several former occasions. The history is as follows. A man, aged 61 years, was suffering from general malaise, pyrexia (temperature $103 \cdot 5^{\circ} \mathrm{F}$.), shivering and pain referred to the back and the right side of the abdomen. Although the respirations were quickened I could find no local symptoms sufficient to account for the illness which was evidently not due to renal calculus. Within a few hours physical signs appeared at the lower part of the right side of the chest and the patient passed through the ordinary course of a rather severe attack of pleuropneumonia of the right lung.

Gentlemen, I think I have said sufficient to impress the practical importance of not neglecting a complete and thorough examination of the chest in all those cases of acute abdominal pain which are sudden in onset, accompanied by 
pyrexia and quickened respirations. You must remember that in such cases the physical signs denoting implication of the contents of the chest may be some hours, not infrequently some days, before developing sufficiently to warrant an exact diagnosis. You will observe that in many of these cases the condition has been that of pleurisy, not pneumonia, but you will find that the physical symptoms, especially at an early stage, are difficult to interpret; they are frequently of hybrid nature; and it may be impossible to distinguish between a simple pleuritis, pleuro-pneumonia, or basic pneumonia.

Influevzal PNeumonia.

The extraordinary variation in the toxic influence-degrees of virulence of an infecting organism-is seen at its best in influenza, and in the influenza bacillus we have an agent profoundly modifying all conditions of pneumonia. The organism has the power to produce per se pneumonia. It also may infect a pneumonia, catarrhal or lobar, which has been caused by the pneumococcus. It would appear as if the two organisms worked together in perfect harmony when infecting the human host. We have clinical evidence that the toxic influence of influenza may also produce a sudden and temporary engorgement of luno tissue, whether of the nature of a simple œdema I cannot say. Writing on this subject in 1894 I made the following remarks :-

I would especially refer to the insidious nature of the onset (influenzal pneumonia) and the rapidity with which large tracts of lun tissue have been implicated in the seizure. In many cases which have personally watched the attack has been of the nature of a lun paresis, and judging from the physical spmptoms the state is one of rapid cedema or passive engorgement. In two instances where an examination was made after death the pneumonia was of this kind and apparently totally distinct in its true pathology from either the lobar or catarrhal form.

The experience of the past ten years has confirmed me in this opinion. I consider that lung symptoms, physically of the pneumonic type, may be due to a rapid engorgement or adema of tissue. The following three cases are of interest and, I think, support such a view.

In the first case a schoolboy, aged 15 years, was seen by me in January, 1900. The lad had come from the seaside where he had been staying with friends who were suffering from epidemic influenza. At 5.P.M. he was considered well, ate a good tea, and after the meal had occupied himself in hanging some pictures. About 7 o'clock he became drowsy and vomited several times. I saw him at 9 P.M. His temperature was $104^{\circ} \mathrm{F}$, the respirations were 45 , and the pulse was running, uncountable. There was a slight hacking cough but no expectoration. On examining the chest tubular breathing could be distinctly heard over the left scapular region. On the following morning the temperature had fallen to $99^{\circ}$, the respirations being 14 , and the pulse 80. Moist sounds were to be heard over the area where on the previous evening tubular breathing was distinct. There was some expectoration which was very slightly tinged. Recovery was without any further incident.

The second case was that of a man of advanced age, whom I saw in consultation with Dr. H. C. Barr in the North of England. On Jan. 13th, 1899, the patient had been out driving and apparently was in normal health. In the evening he complained of feeling unwell and was chilly and without appetite. On the following day he was very drewsy and extremely restless with twitching of the muscles. When seen by me on the third day of illness the symptoms were distinctly cerebral. In fact, he was considered to be suffering from cerebral hæmorrhage. The drowsy condition had deepened into stupor and $I$ had difficulty in rousing the patient. The temperature was $103^{\circ} \mathrm{F}$., the respirations were 45 , and the pulse was 100 ; over the left scapular region the breathing sounds were tubular. On the fourth day the storm had passed, the temperature, the pulse, and the respirations being now normal. There were a few moist râles to be heard where the blowing inspiration had been previously audible. The expectoration, although scanty, was rusty. There were several cases of influenza in the house at the time.

The third of these cases was that of a woman, 67 years of age, who was seen by me on the morning of Dec. 13th, 1899. The patient had gone to bed feeling well. During the night her throat began to feel painful. When I saw her at $10 \mathrm{~A} . \mathrm{Mr}$. there was little to be seen in the throat beyond some injection of the fauces. The glands at the angle of the jaws were slightly swollen. There was headache and general pain was felt about the body. The temperature was $103 \cdot 5^{\circ}$. F. At
6 P.M. the soft palate was much swollen and odematous, the appearance being that of phlegmonous sore-throat. The patient had a bad night, being restless and without sleep, but by the morning the swelling of the soft palate had entirely gone, the temperature being $100^{\circ}$. At 5 P.M. the patient suddenly became delirious, threw off the bedclothes, and was very restless. At 6 P.M. the restless stage had passed into a state of coma and the respirations were now 40 and tubular breathing could be heard at the middle lobe of the right lung. My friend Dr. Andrew Elliot watched the case for me and spent the night in attendance. I do not think that either of us expected the patient to live, but by the morning the temperature had fallen to $97 \cdot 2^{\circ}$ and the respirations to 24 . The patient was dazed and had no recollection of the past 24 hours. There were some moist sounds to be heard in the right lung. Recovery was rapid and complete, the urgent symptoms lasting three days.

It has occurred to me as possible that the state of the mucous membrane of the soft palate might afford an explanation for the anomalous clinical symptoms which were here present. The cedematous engorgement of these parts was so extreme and so evanescent that I could not but surmise that the lesion of lung area might be of the same character. All three of these cases had much the same local physical signs, which were perfectly distinct and easily demonstrable. In each the phenomenon came on with that extraordinary suddenness which, as far as my own experience helps me to express an opinion, is only to be met with in influenza. In each case the illness was accompanied by a profound stupor, the attack passing off as quickly as it had commenced.

In the absence of bacteriological examination and the isolation of the specific microbe of influenza, it is impossible to be actually certain in any individual case whether influenza is the essential factor of the illness, but there are certain well-marked clinical phenomena which, to my mind, justify the assumption that we may be dealing with such a toxic influence. In 1902 there appeared a paper by Dr. J. W. H. Eyre and the late Dr. J. W. Washbourn with the title "Unrecognised Influenza." They preface their clinical remarks by stating that: "As is well known. influenza frequently escapes recognition. This is true not only in mild cases but even in the fatal ones. In making observations upon broncho-pneumonia we have had occasion to examine a number of lungs, both of children and adults, and we have frequently found the influenza bacillus in cases in which the disease was not suspected."

It is next to impossible to carry out a carefully conducted bacteriological examination in every case of doubtful pneumonia or anomalous conditions of pulmonary mischief, so I think it is well to endeavour to differentiate between those cases which are clearly-may I say?-of the old-fashioned type and those in which the toxic influence of the influenza bacillus may profoundly modify the clinical course and symptoms of the pulmonary attack. Personally I attach much importance to irregular fluctuating temperature. If there is one symptom which marks an ordinary lobar pneumonia it is a high, fairly level range of temperature, sustained with but little variation up to the moment of crisis, the pre-critical period depending upon the amount of tissue implication in the inflammatory process. In influenza this does not hold good and we find the temperature fluctuating within extreme limits. A man, aged 26 years, was admitted into the West London Hospital on Dec. 10th, 1904. He had well-marked consolidation of the right base, but he had been up and about his work, though feeling ill for some days before his admission. The temperature was $104^{\circ} \mathrm{F}$., falling on the following morning to $102^{\circ}$ and on the third day to below $100^{\circ}$; on the fourth day rising again to $105^{\circ}$, on the fifth falling three degrees, on the sixth again falling to $100^{\circ}$, but rising during the day to $103^{\circ}$; a critical fall with no further rise occurring on the seventh day after admission. With regard to the range of temperature in any ordinary case of pneumonia most of us would be able to give a fair guess as to the nature of the disease by a glance at the chart, but from the chart before us it would be difficult, if not impossible, to say that it referred to a case of lobar pneumonia. And yet the physical signs were typical of the lobar variety of pneumonia. When the patient was admitted there was dulness over the lower half of the right chest. Over this area tubular breathing and bronchophony were to be heard. There were 


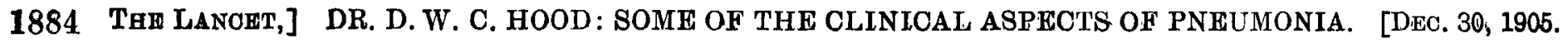

increased vocal fremitus, a high temperature, delirium, and congh with rusty sputa-in a word, all the classical symptoms of a severe attack. But the range of temperature was quite unusual. The chart shows great fluctuations and apart from this there were other symptoms not usually met with in an ordinary attack of lobar pneumonia. The exhaustion was very great, far greater than would be usual in the case of a temperate, healthy young man suffering from an uncomplicated attack. Again, on the third day after admission the chest symptoms were those of acute bronchitis. Moist sounds were to be heard all over the chest. Moist sounds, occurring in the course of lobar pneumonia and affecting the sound lung and accompanied with high temperature, are of extremely ominous significance and betoken great failure of strength. In the case of the patient these symptoms were present for at least 24 hours, the illness terminating in a "crisis" on the seventh day. I have before me as I write the temperature chart of a patient who was taken suddenly ill on the morning of Jan. 11th. Her symptoms were headache, pain in the limbs and back, with extreme gastric irritability, and the temperature was $102 \cdot 4^{\circ} \mathrm{F}$., mounting up to $104 \cdot 2^{\circ}$ in the evening. On the following morning there was a drop of three degrees, but there was now some consolidation of the right lung, the lower lobe being the part affected. In this case, although the physical signs were those of an extensive lobar pneumonia, the temperature during the eight days of illness oscillated between $104.5^{\circ}$ and $99^{\circ}$. The course of the case also differed from that usually seen in lobar pneumonia by the excessive perspiration and a general asthenic condition, the patient dying from exhaustion on the morning of the eighth day of the attack. With lobar pneumonia of an influenzal type the fluctuating temperature is still more marked and a patient may suffer for weeks with constantly recurring attacks of limited broncho-pneumonia, each seizure being accompanied with a rise of temperature. The following case illustrates the point $I$ would enforce. The patient, a man 70 years of age, was suffering from an ordinary attack of influenza with pulmonary symptoms. There were indications of a small patch of lobular pneumonia in the right lung. The local symptoms quickly subsided, the temperature falling, and the general condition of the patient was considered satisfactory. But the ebb and flow of temperature during the fortnight following the initial seizure were sufficient to warrant anxiety. The pneumonic symptoms were but of the slightest. The constitutional disturbance was not severe, the pulse not rising above 84, and the respirations being from 24 to 26 . In such a case the pneumonia might with justice be called migrans. Successively, independent lobules of pulmonary tissue became involved, and lastly a general bronchopneumonia of the hitherto unaffected lung quickly ushered in the final scene. I warn you that with pneumonia of the influenzal type you cannot be too careful in giving your patient the most anxious supervision so long as the pyrexic stage exists. During the first few years of the influenza epidemic one of the most striking facts in connexion with the visitation of that plague was the large number of patients suffering from a form of irregular pulmonary disease.

The mortality of lobar pneumonia is not high and we may have under our care case after case recovering without complication or ever anxiety. How different when the pneumonic seizure is tainted with the poison of influenza. I remember some years ago one of the residents saying to me, quite distressed in his mind, "We are admitting a very large number of cases of pneumonia; they all die." We were at that time in the midst of a severe epidemic of influenza. Case after case of pulmonary trouble of hybrid type was admitted into hospital, the mortality being excessive and quite unusual with that met with in ordinary pneumonia. The toxic influence of influenza is peculiarly depressing, rapidly influencing the vital powers. Old and youns. suffer alike and the asthenic type of the disease is almost akin to that we meet with in diphtheria. The depressed condition seen with pneumonia complicating influenza is, I believe, of the utmost significance when considered from the point of treat ment. I believe it is possible in many cases to distinguish between ordinary pneumocoscal pneumonia and that caused by the influenza bacillus, the main points of difference bein that with the latter disease asthenia and copious perspiration are more common, the range of temperature is not uniformly so high and is far more irregular. The type is often a mixed one and we may find bronchitis superadded or complicating a pneumonia which in its early stage was of a lobar type.
The Modification of the Critical Stage.

Perhaps one of the most striking symptoms of acute lobar pneumonia is the well-known phenomenon of "crisis." Numerous have been the hypothetical explanations of this sudden cessation of an acutely anxious condition of the patient, the accession of calm after, may be, days of distressing illness. We can appreciate the wonder felt and expressed by older physicians on the strange occurrence during the course of a purely inflammatory lesion, a symptom, as expressed by Wilson Fox, "almost peculiar to the disease." But now that we know the essential pathology of pneumonia the occurrence of "crisis" remains no longer a puzzle, no longer can we claim it as being peculiar to the disease pneumonia. We regard it as being the death knell of an infecting organism and an event common to many diseases due to such contaminating influences. The day on which crisis occurs has afforded scope for many expressions of opinion, but I think there can be but little doubt that the leng:th of the illness preceding the sudden cessation of pyrexia, whether measured by hours or days, is directly referable or proportioned to the amount of the inflammatory lesion, in other words, to the amount of lung tissue involved in the process. There cannot be a question that a very slight attack of pneumonia, in which only a small portion of lung is involved, is of shorter duration than when the entire lung is the subject of the inflammatory lesion. Between the two extremes we find every gradation and we are bound to recognise the existence of a one-day pneumonia as much as we do one that exists for ten days or more. As practical physicians our attention is called to those cases in which a crisis does not occur, and it is well to consider what may be the causes which may interfere with, or prevent, that sudden fall of temperature so typical of an ordinary attack of pneumonia. Without being exhaustive, I would name the following complications as impeding or entirely preventing crisis. Pericarditis occurring during the course of pneumonia will keep up pyrexia and will mask the critical fall. The same may be said of pleurisy, especially when purulent. If during an attack of pneumonia limited to one side of the chest the other lung should become affected the critical stage is delayed and, as far as my own experience serves me in expressing an opinion, the phenomenon of crisis is hardly so pronounced. Should, however, there be an interval between the two separate infections there may be two well-marked critical falls of temperature. Should a lobar pneumonia be followed by bronchitis or catarrhal inflammation-an event which occasionally happens in the case of children-the crisis is absent. The influenza bacillus may influence the critical stage, but in what distinct manner I cannot say. In some cases of influenzal pneumonia a crisis is as definite and well marked as in ordinary lobar pneumonia, while in another case with practically the same physical conditions crisis does not occur. With patients the subject of lobar pneumonia in whom there is no critical fall, the temperature oscillating and of almost hectic type, it is important to examine carefully the chest with a view of ascertaining the existence of tluids; in many such cases an empyema is the disturbing infuence, and it is well to recognise how easily we may be deceived by looking upon a lung as still being in a state of consolidation, whereas resolution has insidiously taken place and the chest has as insidiously filled with pus. If in what is apparently a pure uncomplicated attack of acute lobar pneumonia the critical stage is absent and resolution does not take place within reasonable time it behoves us to examine the chest with the most conscientious care. Of all the secondary conditions leading to the absence of crisis none is more common than post-pneumonic empyema, and if we are not fully alive to this complication it is easy to drift complacently into error with regard to the true pathology of the case. The fever does not fall, the lung does not resolve; day after day it is watched and the dulness and tubular breathing remain. Here the attack has commenced with all the characteristic features of acute pneumonia; there has been no symptom denoting that with the subsidence of the pneumonic inflammation there has been a gradual steady effusion poured out into the corresponding pleural cavity. I think that when we examine such cases and find the supposititious pneumonic consolidation melt away and fluid taking its place we are apt to feel uncharitable towards our neighbours. In clinical medicine it is so very easy to be wise after the event. And the patient we examine, perhaps for the first time, and in whom we find evidence of fluid when a solid lung has been supposed 
to exist, may have been treated with the greatest care and attention; in fact, it is often with those patients we are in the habit of seeing most frequently that such an error of diagnosis is most apt to occur. The development of the effusion may be insidious and without any special symptoms ; hence the transition from consolidated lung to pleural effusion is not recognised. But, even putting aside the primary history of such a case, the differential diagnosis between solid and fluid is not always so easy as it would appear; physical signs occasionally prove very misleading. Dr. Thomas Addison, in his twenty-seventh aphorism, writes: "When serous effusion is very considerable, giving rise to unequivocal bronchophony, tubular breathing, want of resonance, and vocal vibration, physical examination has repeatedly led to a mistaken belief that these signs resulted from a pneumonia or other consolidation of lung," and I have no hesitation in affirming with equal confidence that similar mistakes may occur even if the effusion be purulent. Of course, it is quite possible for the preceding pneumonia to run its ordinary normal course and terminate by crisis, the effusion following upon the pneumonic process, but my remarks have special reference to those cases in which there is no crisis, but where the temperature either ebbs and flows or, as is sometimes the case, gradually falls to normal or but little above, the chest meanwhile showing no sign of improvement and the dulness remaining.

The following is a typical example of a pneumonia followed by purulent effusion. I saw a patient in consultation who had been suffering from pneumonia for about a month. The initial attack had been severe and the symptoms usual and unequivocal. The patient was a large-framed, strongly-built man, 48 years of age. His medical attendant considered that the lung had not "cleared up," and certainly when the chest was examined the physical symptoms were strongly confirmatory of that opinion. The lesion was on the left side, which was dull, but not to that extent usually present when fluid is in quantity. Vocal resonance and tubular breathing were both very distinct. The cough was loose and constant, the temperature ranging from $100^{\circ}$ to $103^{\circ} \mathrm{F}$. The patient had a large deep chest and it was difficult to localise exactly the apex beat of the heart. I considered that the sounds were best heard to the right of the sternum. From the irregularity of the temperature, from the fact that there had been no crisis, and from my belief that the heart was displaced, I felt justified in suggesting an exploration, the result being that two pints of creamy pus were withdrawn by aspiration and the patient made a rapid recovery.

The following is a case of much the same class. A woman, 26 years of age, was admitted into the West London Hospital on Jan. 24th, 1894 . She had been ill for three weeks, having suffered from an attack of lobar pneumonia accompanied with the usual symptoms. There had been no crisis, although the temperature gradually fell; on admission it was but slightly raised and a few hours after the patient was placed in bed it was recorded as being subnormal. The principal symptom was dyspncea; the patient was in the sixth month of pregnancy. The left chest was absolutely dull, the dulness extending to the right beyond the middle line of the sternum. Posteriorly, tubular breathing and whispering pectoriloquy were well marked; the heart, much displaced, was apparently beating below the right nipple. "The patient was in much distress, the respirations being 66 . Aspiration was immediately performed, rather more than two pints of sweet pus being removed. With regard to diagnosis the case presented but little difficulty, yet there were numerous points of interest. The temperature contra. indicated purulent effusion; there had been no rigor or any other symptom indicative of pus. The total duration of illness had been short; the early initial stage was that of pneumonia-a pneumonia which drifted by degrees into a vast purulent effusion with so much tension and disturbance of part that I thought it best to remove the fluid by degrees. I aspirated the chest on three occasions and subsequently a free opening was made, a portion of rib being excised, the patient making a complete recovery.

Cases in which an initial pneumonia has been followed by effusion of a purulent character are so important from the point of view of treatment that $I$ do not scruple to refer to them. I merely touch upon the most salient points of each case.

A girl, 18 years of age, was admitted into the North-West London Hospital under my care, the history of attack being that she had caught cold and had had a severe shivering fit. I saw her on the afternoon of the day of her admission; hex condition was pathognomonic of acute pneumonia. The respirations were 46 , the temperature was $104^{\circ} \mathrm{F}$., and the pulse was 130 . The physical symptoms were but poorly marked; there were some sharp, fine crepitations over the base of the left lung but no pain or stitch on breathing. On the following day crepitation could be heard np to the angle of the scapula, the base being now dull. From this time the patient was severely ill, so ill that an accurate, careful examination of the chest was impossible ; the lower part of the right lung became implicated and the constitutional disturbance extreme. Seven days after admission the physical conditions were as follows: on the left side were tubular breathing and bronchophony; the apex beat of the heart could not be felt but the sounds were loudest at the left of the sternum. There had been no shivering or crisis, the temperature gradually falling. The general condition of the patient improved and she pronounced herself as feeling much better; nevertheless, the breathing continued rapid and embarrassed. At this period the balance of symptoms was in favour of the presence of fluid, though a careful physical examination failed to support that view. A few days later the girl was seen again; she expressed herself as being much more comfortable; she had slept well and had taken food with appetite. The temperature was now normal but both pulse and respirations were still rapid. Cough was frequent but unaccompanied with any expectoration; it had assumed an almost paroxysmal character. At this interview I felt certain that the heart was displaced. An aspirator withdrew a pint of sweet pus. On the following day the chest was opened and two pints of pus were removed, the subsequent history being without incident.

Another case bearing on the matter occurred in my practice at the West London Hospital in March, 1888. A male patient was admitted suffering from pneumonia of ordinary intensity: there was nothing irregular in the clinical course and the normal physical signs were present. There was a rather free expectoration of rusty and bloodstained sputum. On the seventh day after admission the general state of the patient was satisfactory; the attack had run an ordinary course, with the exception that there had been no crisis. The temperature had gradually fallen and on the morning of this day was $99^{\circ} \mathrm{F}$. The percussion note at the lower part of the right chest was toneless-that is, was more dull than is usually the case from pneumonic consolidation. The cough was irritating and paroxysmal; the ordinary cough of pneumonia with the viscid rusty sputum had changed to one without any expectoration, but was severe and recurring in paroxysms. A hypodermic syringe revealed the presence of fluid; a small quantity, amounting to about four ounces, was withdrawn; it proved to be pus and was sweet. On the following day the temperature was normal, the cough had entirely ceased, and within a few days the patient was discharged well.

Another interesting case came under my care in the West London Hospital. The patient, a finely built, muscular young fellow, was admitted for pneumonia, the primary symptom being a rigor. The constitutional symptoms were so pronounced that a very careful examination was impossible. During the first week the illness was complicated with violent maniacal delirium and it was only with difficulty that the patient could be kept in bed. There was an abundant crop of herpes on the lip, the sputum being viscid and rusty. The clinical features were purely those of a severe lobar pneumonia. The delirious stage was followed by a sharp attack of pericarditis, which again prevented a careful examination, and it was not until the patient had been a fortnight in hospital that we were able to estimate the amount of chest mischief. Briefly, the physical signs were as follows : the right chest was dull, tubular breathing and bronchophony were well marked; the general strength of the patient had improved; the heart beats were in the normal position. The liver was not depressed and vocal fremitus could be felt over the dull area. The clinical evidence was against the presence of fluid. A week later the physical signs were much the same, the general state of the patient being satisfactory; the temperature falling gradually was now but slightly above normal. The cough, however, had entirely altered in character: it was peculiar, coming on with storm-like severity, suggesting an attack of whoopingcough; there was no expectoration. A hypodermic syringe
detected pus; three pints were removed; subsequently a 
1886 The Lanoet,] DR T. WILSON: MEDIOAL TREATMENT OF UTERINE FIBROIDS, ETC. [Dec. 30, 1905.

free opening was made and the patient made a complete recovery.

I would specially invite your attention to the peculiarity of the cough which may often attend these residual effusions. I have frequently had occasion to remark it and now look upon it as a valuable auxiliary symptom towards the diagnosis of fluid. It is essentially paroxysmal, commencing after movement, and is not usually followed by expectoration. I believe that such a cough following upon the symptoms of an ordinary pneumonia is pathognomonic of the presence of fluid; the fluid may be in very small quantity. In one case, that of a child, four drachms of pus were aspirated; the cough, which was akin to pertussis, ceased upon the withdrawal of the fluid. We frequently meet with cases in which a pneumonia complicated by pleuritis is followed by abnormal dulness of the affected side of the chest. We explore the pleural cavity and no fluid is found. In many such instances the explanation is doubtless a thickened pleura.

Occasionally pneumonia may terminate by crisis and be followed by effusion, but without any subsequent rise of temperature. A case in point is the following one of a man, aged 30 rears, who was admitted into the West London Hospital under my care on April 13th, 1892. The symptoms were those of an ordinary attack of lobar pneumonia of the left base reaching to the angle of the scapula. The physical signs were definite and of the usual character. The patient had been ill for four days before admission. On the morning following the day of admission the temperature fell to below normal but on the evening of that day it rose again to $104^{\circ} \mathrm{F}$. At this time it was found that there were symptoms of inflammatory mischief at the base of the right lung. The pyrexic stage was of but very short duration, lasting about 36 hours, and followed by a second critical fall. From this time the temperature did not rise again but there were unequivocal symptoms of effusion which necessitated aspiration on the tenth day after the second crisis, the interesting point being that although there was a large serous pleuritic effusion there was no accompanying rise of temperature. I must warn you that the contrary is much more usual and you will find that a slight rise of temperature is concomitant with effusion even if that effusion be of serous tluid.

Before leaving the subject of post-pneumonic empyema I wish to mention a practical point which happily occurs but rarely, but when present may very seriously influence our opinion and diagnosis. I allude to those cases where an effusion in the left chest is not associated with displacement of the heart, the heart being bound down by adhesions due to a former pleurisy. A very striking instance of this occurred in my practice last year. The patient, a man aged 60 years, passed through a sharp attack of pleuro-pneumonia which was followed by dulness on the left side. But opposed to the view that fluid was the cause of this physical symptom was the fact that the apex beat of the heart was well marked below the left nipple; subsequent events proved, however, that the heart in this case must have been twisted over, being probably bound down by adhesion, for in spite of the fact that four pints of pus were withdrawn from the chest the pulsation immediately below the nipple was present from the commencement of the effusion until the time it was withdrawn. These anomalous cases are referred to in an article by Dr. Schroetter in the sixth volume of Ziemssen. I can only recall one case occurring to me among hospital patients. When such anomalous physical conditions do occur the heart, instead of being pushed to the right without changing the relative positions of base to apex, is tilted to the right, so that there may appear two points of pulsation-one at the epigastrium, or to the right of the sternum, and the other due to pulsation of the right ventricle, at or about the normal position of the apex beat. There is, in fact, a spurious apex beat at or below the left nipple due to pulsation of the right ventricle.

Bradford Medico-Chirurgical Society.The annual dinner of this society was held on Nov. 28th at the Midland Hotel, when Professor Frank Thomas Paul delivered a lantern lecture on the Use of the Microscope as an Aid to Diagnosis. There was a large attendance of members and friends.

\section{THE MEDICAL TREATMENT OF UTERINE FIBROIDS AND ITS LIMITATIONS. ${ }^{1}$ \\ By THOMAS WILSON, M.D. LoNd., M.CH. Birn., F.R.C.S. ENG.}

BEFORE considering the treatment of uterine fibroids it is especially necessary to study the natural history of the affection for it is well known that the tendency towards impaired health or a fatal issue is not inevitable in this disease as it is in ovarian cysts and many other affections for which the aid of surgery is invoked. The uterus is the organ of all others most frequently affected by the growth of tumours, both simple and malignant. As regards simple tumours Bayle in the early years of last century stated that 20 per cent. of all women over the age of 35 years have uterine fibroids. 'The truth of this startling assertion was proved for me by Dr. M. Randall and Dr. Stuart Macdonald by a series of observations which are briefly set out in a paper published in the Birmingham Medical Rerien for November, 1904. The statement refers merely to the presence of fibroids that can be recognised in the post-mortem room and the great majority of the tumours are small, varying from the size of a pea to that of a walnut. If now we consider the number of women suffering from the effects of fibroids, as compared with the number of those in whom fibroids are merely present, we find that the rate is very much lower; thus, for instance, in about 8000 cases of diseases of the female generative organs I made the diagnosis of fibroids in 274 cases, or 3.5 per cent., the frequency being almost exactly equal to that of uterine cancers in the same series. If we compare the clinical rate thus shown with the pathological frequency it appears certain that the enormous majority of uterine fibroids give rise either to no symptoms at all or merely to slight or transient symptoms that do not lead to the recognition of the disease during life--in fact, most uterine fibroids are of no more importance clinically than warts or nævi. In a certain proportion of women, however, uterine fibroids do give rise to trouble more or less serious and in considering the treatment to be pursued it may be useful at the outset shortly to enumerate the graver symptoms and conditions to which fibroid tumours give rise, those effects, namely, that may justly be held to demand the employment of surgical methods.

Operations for uterine fibroids have become progressively more successful during the last 15 years until now the mortality following them is as low as that of ovariotomy. In the last 56 total abdominal hysterectomies for fibroid in the obstetric department of the Birmingham General Hospital and in my private practice the mortality has been $7 \cdot 1$ per cent. The indications for operation vary widely in the practice of different operators ; some are of opinion that radical surgical treatment should be undertaken in every case in which the presence of a fibroid or fibroids is determined; while others, myself among the number, contend that since the operation for their removal is not rarely followed by inconvenient symptoms of long duration, radical surgical means should only be employed when the life or continued good health of the patient is threatened. It is, of course, obvious that if every case diagnosed were operated upon the statistical results would nowadays be excellent, but it has to be remembered that brilliant operating is not necessarily good treatment.

In the 274 cases just mentioned there were 26 fibroid polypi up to the size of a cocoanut. Leaving these out of consideration there remain 248 cases of fibroids, among: which 74 were treated by radical operations and chiefly by abdominal hysterectomy. An analysis of the symptoms in these 74 cases shows that operation was undertaken in 29 instances for a tumour already of considerable size or rapidly growing or associated with some severe symptoms, such as pain or menorrhagia ; in 14 the indication was excessive or persistent uterine hæmorrhage ; in 13 the chief symptom was severe or incapacitating pain ; and in six others there had been pelvic incarceration of the tumour leading to retention of urine. The remaining cases included two operations undertaken primarily for ovarian cyst, one for tubal gestatior, one

1 A paper read on Oct. 31st, 1905, at the annual meeting of the Shewsbury and Mid-Wales branch of the British Medical Association. 